\title{
A Contribuição da Teoria Geral dos Sistemas e do Pensamento Complexo para o entendimento dos sistemas de inovação nacional/regional
}

\author{
Vivian Costa Brito ${ }^{1}$ \\ Ronaldo Leão de Miranda ${ }^{2}$ \\ Gilberto Friedenreich dos Santos ${ }^{3}$ \\ Cristiane Mansur de Moraes Souza ${ }^{4}$
}

\begin{abstract}
RESUMO
A complexidade dos sistemas de inovação nacional e regional pode ser analisada a partir da teoria geral dos sistemas e do pensamento complexo aplicado a um dado contexto territorial. Nessa perspectiva, o objetivo deste estudo é compreender qual a contribuição da teoria geral dos sistemas e do pensamento complexo para o entendimento dos sistemas de inovação nacional/regional. Diante disso, tem-se que a teoria dos sistemas auxilia no entendimento das unidades e as alterações escalares (micro, meso e macro) entre subsistemas que compõe os sistemas de inovação, suas relações entre si, que caracteriza sua vulnerabilidade ou resiliência. Portanto, as experiências apresentadas sobre sistemas de inovação refletem a realidade dos países ao Norte em melhor contexto de desenvolvimento do que os países ao Sul. Por isso, devem ser analisadas como referenciais epistemológicos apenas para suscitar questionamentos e possibilidades diante do contexto dos países ao Sul. Pois, há indicações que para atingir tais resultados é preciso superar as desigualdades, exigindo políticas orientadas para a constituição do sistema.
\end{abstract}

Palavras-chave: Teoria Geral dos Sistemas. Pensamento complexo. Interdisciplinaridade. Sistema de Inovação Nacional/Regional.

\section{The Contribution of the General Theory of Systems and Complex Thought to the Understanding of National / Regional Innovation Systems}

\begin{abstract}
The complexity of national and regional innovation systems can be analyzed from the general theory of systems and complex thinking applied to a given territorial context. In this perspective, the objective of this study is to understand what the contribution of general systems theory and complex thinking is to the understanding of national / regional innovation systems. In this way, it is possible that system theory assists in the understanding of the units and the scalar changes (micro, meso and macro) between subsystems that make up the systems of innovation, their relations among themselves, that characterize their vulnerability or resilience. Therefore, the experiences presented on innovation systems reflect the reality of the countries to the North in a better context of development than the countries to the South. Therefore, they should be analyzed as epistemological references only to raise questions and possibilities in the context of the countries to the South For there are indications that in order to achieve these results it is necessary to overcome inequalities, requiring policies oriented towards the constitution of the system.
\end{abstract}

Keywords: General Theory of Systems. Complex thinking. Interdisciplinarity. National/Regional Innovation System.

\footnotetext{
${ }^{1}$ Doutora em Desenvolvimento Regional, Mestre em Análise Regional, licenciada em Geografia e graduada em Turismo. Atualmente é docente do Colégio SESI Internactional Trilingue e da SEED do Paraná. Atua como consultora na área do Planejamento Urbano/Regional, no Turismo e na Hotelaria, com produção técnica de diagnóstico socioeconômico, planos de ação, roteiro ecoturístico, censo, projeto pedagógico e plano de desenvolvimento turístico. ${ }^{2}$ Doutor em Ciências Contábeis e Administração (FURB). Mestre em Desenvolvimento Regional (FURB). Bacharel em Administração (URI) e Bacharel em Ciências Contábeis (UNESA). Professor Substituto do Instituto Federal de Educação, Ciência e Tecnologia do Mato Grosso (IFMT) Campus Várzea Grande.

${ }^{3}$ Geógrafo, Mestrado em Geografia e Doutorado em Geografia. Atualmente é professor e pesquisador do Programa de Pós-Graduação em Desenvolvimento Regional, da Universidade Regional de Blumenau (FURB).

${ }^{4}$ Arquiteta e Urbanista, mestre em Urban Design Ma (Oxford Brookes University) e doutorado em Interdisciplinar em Ciências Humanas. É pesquisadora produtividade do $\mathrm{CNPq}$, professora do programa de pós-graduação em Desenvolvimento Regional, do Curso de arquitetura e urbanismo e do curso de engenharia civil, ambos da Universidade Regional de Blumenau (FURB). Realizou estágio sênior em ciências ambientais (bolsa CAPES) na Washington State University, Pullman, WA, EUA. Email: mansur@furb.br
} 


\section{INTRODUÇÃO}

Cada vez mais, a inovação é concebida em um contexto de convenções, regras e complexidade (DIAS; PEDROZO; DA SILVA, 2014). Neste contexto, se faz necessária a compreensão das estruturas multinível (micro, meso e macro), para assim, aprofundar a discussão sobre complexidade no que tange a inovação. As características da complexidade, como não linearidade, elementos multiníveis, interações, trajetórias, emergência e sistemas dentro dos sistemas, têm sido observadas em várias publicações sobre inovação (VAN DE VEN et al., 2007; GARUD et al., 2011; DIAS; PEDROZO; DA SILVA, 2014). Além disso, a inovação é a força motriz do desenvolvimento econômico (SCHUMPETER, 2004; COOKE, 2011).

A inovação tem sido objeto de interesse de diferentes disciplinas das áreas humanas e sociais, como economia, administração e desenvolvimento regional. Seu estudo numa perspectiva geográfica passa a emergir quando surgem sistemas de inovação aplicados a um dado contexto territorial, quer seja a escala atribuída nacional, ou escala regional. Para Asheim e Gertler (2009), os sistemas de inovação em escala subnacional desempenham um papel fundamental na produção e reprodução do espaço. Neste contexto, é importante destacar que uma série de condicionantes é necessária para a formação de um sistema de inovação. Cabe destacar a capacidade institucional no interior do sistema, a partir dos aspectos financeiros, de aprendizagem e as "culturas" produtivas.

Ainda, os modos de interação entre os diversos atores, bem como os fluxos de conhecimento e informação. Nas últimas décadas, tem havido um crescente interesse pelo desenvolvimento de pesquisas e formulação de políticas públicas por meio da abordagem de sistemas de inovação. No entanto, conforme evidenciado na literatura acadêmica, faltam estudos sistemáticos, cronológicos e sintetizadores que indiquem como essa área tem evoluído ao longo do tempo (URIONA-MALDONADO; DOS SANTOS; VARVAKIS, 2012; SCHOT; STEINMUELLER, 2018). Assim sendo, este artigo pretende compreender qual a contribuição da teoria geral dos sistemas e do pensamento complexo para o entendimento dos sistemas de inovação nacional/regional. A partir do delineamento do objetivo, este estudo busca responder a seguinte questão: como a teoria geral dos sistemas e do pensamento complexo pode servir para o entendimento dos sistemas de inovação nacional/regional?

Metodologicamente, trata-se de uma pesquisa descritiva em relação a seu objetivo, quanto aos procedimentos pode ser considerada como bibliográfica, e qualitativa em relação à abordagem do problema, sendo analisada por meio de análise de conteúdo (GIL, 2017). No caso de ser um trabalho descritivo e qualitativo, segundo Yin (2005), representa a estratégia ideal quando se colocam questões do tipo "como" e "por que".

Portanto, o artigo está divido em cinco partes, a começar por esta introdução. Logo, apresentam-se as abordagens sobre a teoria geral dos sistemas e sua aplicação na análise do desenvolvimento regional. De modo a complementar estas abordagens, procura-se entender a aplicação do pensamento complexo a partir da abordagem interdisciplinar, para então tratar dos sistemas nacionais de inovação e sistemas regionais de inovação no contexto territorial e por fim, aborda-se, as considerações finais.

\section{A TEORIA GERAL DOS SISTEMAS E SUA APLICAÇÃO NA ANÁllSE DO DESENVOLVIMENTO REGIONAL}

A complexidade das relações produtivas e de inovação, presentes em dado contexto de desenvolvimento regional podem ser interpretados pelo modelo sistêmico. Esse modelo é originado da teoria geral de sistemas desenvolvida pelo biólogo Ludwing von Bertalanffy (2010) na primeira metade do século XX. 
Entretanto, Capra e Luisi (2014, p. 117) apontam que:

[...] vinte a trinta anos antes que Bertalanffy publicasse os primeiros artigos sobre sua teoria geral dos sistemas, Alexander Bogdanov (1873-1928), médico pesquisador, filósofo e economista russo, desenvolveu uma teoria sistêmica de igual sofisticação e igual âmbito, a qual, infelizmente, ainda é amplamente desconhecida fora da Rússia. Bogdanov deu à sua teoria o nome de 'tectologia, do grego (construtor), que pode ser traduzido como "ciência das estruturas. O principal objetivo de Bogdanov foi esclarecer e generalizar os princípios de organização de todas as estruturas vivas e não vivas.

Em seguida, Capra e Luisi (2014), afirmam que objetivo de Bogdanov era elaborar uma ciência universal da organização. Explicam que há uma aproximação conceitual entre a forma organizacional como totalidade de conexões entre elementos sistêmicos de Bogdanov e a acepção atual de padrão de organização empreendidas por eles. O sistema para Bertalanffy (2010, p. 127), funciona "como complexo de componentes em interação, conceitos característicos das totalidades organizadas tais como interação, soma, mecanização, centralização, competição, finalidade, etc".

Nesse mesmo sentido, Miller (1965), define o sistema como um conjunto de unidades com relações entre si e o seu grau de organização permite que assuma função de um todo que é maior que a soma de suas partes. O eixo central dessa teoria é de uma tendência para integração de elementos físicos ou concretos, e, de elementos abstratos ou conceituais. Os elementos físicos são compostos por objetos e coisas concretas. Os elementos abstratos estão no plano das ideias, dos conceitos. A integração só é possível em função da natureza fluida do sistema, na qual há interação e troca entre os sistemas abertos em seus mais diversos elementos presentes no ambiente (BERTALANFFY, 2010).

O sistema é definido por Thornes e Brunsden (1977), como um conjunto de objetos ou atributos de suas relações, que se encontram organizados para executar uma função particular (CHRISTOFOLETTI, 1979). Assim sendo, ao receber uma entrada (input) no sistema, os objetos ou atributos estabelecem relações com os demais em seu interior resultando em alterações, e em um dado momento ocorre a saída (output). Emerge então uma reflexão: toda entrada corresponde a um tipo de saída.

Vale (2012, p.91), em seu estudo relaciona a Teoria Geral dos Sistemas com a geografia, e apresenta uma definição de sistemas ao inferir "inicialmente, que os sistemas devem ter suas partes componentes, denominadas unidades, ou elementos, ou ainda, componentes, as quais devem encontrar-se inter-relacionadas, dependentes umas das outras, através de ligações que denunciam os fluxos". Conforme explicita na Figura 1:

Figura 1: Representação esquemática de um sistema, assinalando os elementos (A, B, C e D) e suas relações, assim como o evento entrada e o produto final
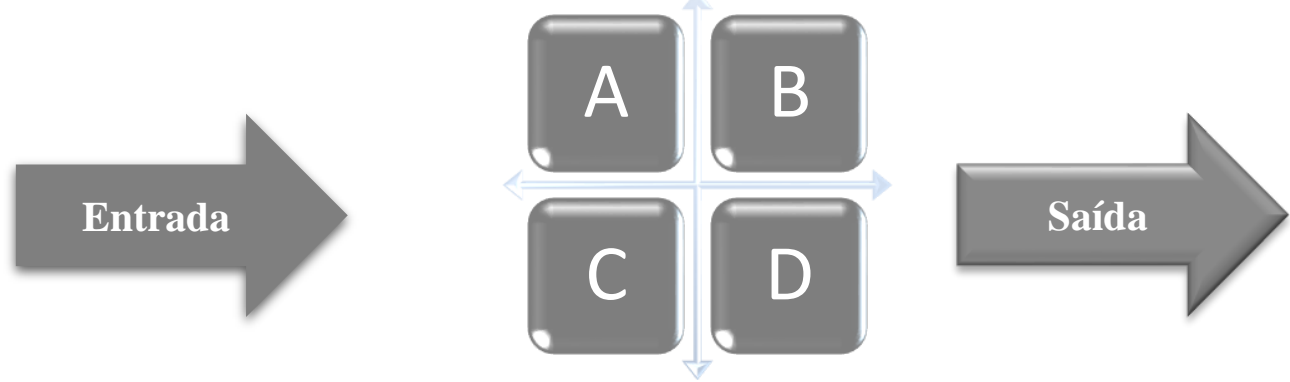

Fonte: Baseada em Christofoletti (1979). 
Destarte, Vale (2012), chama atenção de que as unidades ou elementos apresentam atributos, ou qualidades que as diferenciam diante das demais e que isso produz determinado efeito no sistema. Deste modo, a seleção dessas qualidades pode servir para auxiliar no entendimento das partes do sistema (CHRISTOFOLETTI, 1979). A aplicação deste modelo ao contexto do desenvolvimento regional e a formação de sistemas de inovação regionais deve ir além da afirmação de Davidson (1983, p. 23):

[...] se as leis dos sistemas biológicos - que regem os processos como crescimento e adaptação - podem ser aplicados às áreas além da biologia; e se a lei da gravidade é igualmente aplicável às maçãs e aos planetas; e se a lei da probabilidade se aplica igualmente à genética e aos seguros de vida, então as leis dos sistemas biológicos, bem poderiam ser aplicáveis à psique humana, às instituições sociais, e ao conjunto global da ecosfera.

Destarte, não se pode considerar o sistema social como equilíbrio constante, há que conceber que está vinculado aos desequilíbrios do sistema econômico, das interações sociais que se dão em função de processos culturais que criam particularismos, e por isso atuar numa perspectiva biocêntrica tem sido extremamente conflituoso ao longo do século XX e XXI. Isso significa imbricar as disciplinas de economia, sociologia, ecologia, geografia, dentre outras. Assim sendo, com caráter de pesquisa que ultrapassa os limites das áreas disciplinares, a abordagem teórico-metodológica do desenvolvimento regional compreende estudos interdisciplinares. Esse viés tem nos estudos da problemática ambiental sua principal contribuição.

\section{O PENSAMENTO COMPLEXO E A PRÁXIS INTERDISCIPLINAR}

Segundo Novo Villaverde (1997), a problemática ambiental, sob a perspectiva da Educação Ambiental, é um ponto central para se compreender a aplicação de modelos e metodologias vinculadas ao pensamento complexo e a práxis interdisciplinar. O contexto contemporâneo da problemática ambiental, circunscrito historicamente ${ }^{5}$ por diversos fenômenos de caráter global, encontra-se complexo e policêntrico, cercado por conflitos de interesses entre os atores e conflitos destes com o meio ambiente que não podem ser tratados como fenômenos isolados.

Novo Villaverde (1997), institui como solução para a sustentabilidade a necessidade de mudanças em âmbito econômico. Mudanças estas, de bases tecnológicas, de maior eficiência no sistema de gestão, bem como nos esquemas de pensamento e de nossas formas de ação, com um novo enfoque crítico e inovador de educação. Alude que é preciso pensar sistematicamente, com a integração dos subsistemas em equilíbrio, numa perspectiva de solidariedade intra e inter gerencial do uso dos recursos da terra. Segundo Novo Villaverde (1997), a crise se revela como oportunidade para mudança, que pode culminar em novos modelos de pensamento e ação que deve seguir por meio da sustentabilidade, baseado em modelos descentralizados, que tentam emergir por via da auto-organização.

A solidariedade sincrônica e diacrônica são elementos indispensáveis no processo. Destarte, sugere uma nova concepção de progresso definia como uma ampliação da consciência e da solidariedade no qual se cria o improvável (NOVO VILLAVERDE, 1997). Seguindo a linha de pensamento acima, Capra e Luisi (2014, p. 118), citam Bogdanov ao enfatizar:

um avanço da consciência e da solidariedade em que se constrói o improvável

\footnotetext{
${ }^{5}$ Intensifica-se no século XX, numa sociedade baseada na produtividade, no benefício a curto prazo e na ideia de progresso indefinido.
}

Revista Desenvolvimento Socioeconômico em debate v.7 n.2 (2021) 
de que a tensão entre crise e transformação tem importância central para a formação de sistemas complexos. [...], Bogdanov mostrou como a crise organizacional se manifesta como uma ruptura do equilíbrio sistêmico existente e, ao mesmo tempo, representa uma transição para um novo estado de equilíbrio.

Nesse contexto, Novo Villaverde (1997), suscita que algumas posições inovadoras foram traçadas e outras devem ser enfrentadas. Do ponto de vista teórico vinculam-se os problemas ambientais com a questão ética, tratado por Morin (1991), que atrela a mesma com a ação, pois compreende que ação e pensamento se interpenetram. Há necessidade de se impulsionar os modelos científicos emergentes, que compreendem os limites do conhecimento em poder medir e prever tudo. Simultaneamente, desenvolver e implementar autênticos modelos de desenvolvimento sustentável, a fim de equilibrar o ecossistema global, sistema mundo.

Metodologicamente, Novo Villaverde (1997), propõe que sejam feitos estudos explicativos e não meramente descritivos, considerando os aspectos subjetivos do olhar do pesquisador, que passa a fazer parte da construção do conhecimento e interpretação da realidade, da elaboração de hipóteses, no desenvolvimento da pesquisa e inclusive nos resultados. Numa mesma abordagem, Garcia (1994), não parte de uma concepção pluri ou multidisciplinar. Entende que o escopo de análise desse campo é limitado quando se aborda a problemas locais circunscritos, de maior complexidade, que para isso a abordagem interdisciplinar é melhor aplicável.

Por seu caráter heterogêneo e de interdependência impede que se inclua ao mesmo uma análise setorial dos elementos. Garcia (1994), exemplifica que a partir de estudos setoriais de propostas de políticas alternativas para o desenvolvimento sustentável aplicada a uma região esse modelo se torna fragilizado. Deste modo, Garcia (1994), vai distinguir o conceito de multi ou pluridisciplinaridade e interdependência. Este último chamará de "investigación interdisciplinaria" (GARCIA, 1994, p. 67). Constrói o sentido de complexo aplicado a sistemas, sendo este vinculado com as relações entre sistemas e sua interdependência. Atribui a disciplinaridade uma condição necessária, mas que precisa ser repensada para que haja um avanço para ações interdisciplinares, pois inter significa entre disciplinas. Parte de uma concepção de interdisciplinaridade que busca sua acepção a partir de seu objeto de estudo e sua forma de ser analisado.

Novo Villaverde (1997), ao propor uma visão de pensamento complexo critica as abordagens reducionistas extremamente localistas, "pois es imposible atajar los problemas con reducidas soluciones locales, salvo que estas sean la expresión de movimientos renovadores de amplio alcance y difusión" (NOVO VILLAVERDE, 1997, p. 26). É preciso pensar globalmente e atuar localmente. Para isso, sugere que seja feita uma análise com base no enfoque sistêmico, que considere os complexos mecanismos que regem a ordem dos sistemas. "La complexidad de los sistemas ambientales es un reto a nuestra propria capacidad para complejizar el piensamento y la accion sobre el medio" (NOVO VILLAVERDE, 1997, p. 26).

Os estudos integrados de um sistema exigem marcos epistêmicos, conceituais e metodológicos compartilhados. Entretanto, essas bases conceituais e metodológicas precisam ser aclaradas. As universidades, segundo Garcia (1994), não têm assumido esse papel de formar investigadores, pois trazem um conhecimento fragmentado. Para essa concepção de investigação interdisciplinar, sugere uma reformulação do interior de cada disciplina.

A crítica postulada por García (1994), é extremamente coerente, pois de um lado encontramos a perda de atratividade do conhecimento científico tal qual abordado na universidade de modo estritamente disciplinar, sem conseguir expressar respostas, nem formular questões que possam atinar para as problemáticas que se apresentam no mundo atual. A interdisciplinaridade surge como uma reação à especialização da ciência contemporânea, modelo que fragmenta os problemas da realidade e que dificulta a se chegar à síntese, tão cara à interpretação da realidade complexa. Critica o modo como as disciplinas criaram seus campos de estudo e as pontes estabelecidas entre elas, a exemplo da biofísica que não aproxima da interdisciplinaridade. 
Expõe a importância de se conhecer um problema específico como campo de investigação, dando valor a especialidade. Alude que formações somente interdisciplinares baseadas em múltiplas disciplinas agrupadas, corre o risco de não formar investigadores que consigam fazer uma síntese integradora. Incita que não existem pessoas interdisciplinares, mas equipes pluridisciplinares que atuam de modo interdisciplinar. Esta concepção esclarece quanto aos limites da interdisciplinaridade e confere o valor da disciplinaridade na execução de tal projeto. Em função disso propõe uma reformulação de entender interdisciplinaridade como entre disciplinas.

Garcia (1994), apresenta as características do estudo de um sistema complexo a partir de três fontes: do objeto de estudo, do marco conceitual e dos estudos disciplinares. O objeto de estudo não é reduzível a fenômenos que pertençam a uma só disciplina. O marco conceitual envolve a base teórica em que os investigadores identificam, selecionam e organizam os dados e a realidade a ser analisada. E os estudos disciplinares são os recortes dessa realidade complexa, abordados desde uma disciplina específica. Nesse sentindo, Garcia (1994, 74), apresenta a definição de complexo:

Las interrelaciones entre los componentes, cuyas funciones dentro del sistema no son independientes. El conjunto de sus relaciones constituye la estructura, que da al sistema la forma de organización que le hace funcionar como una totalidad. De aquí el nombre de sistema.

Destarte, Novo Villaverde (1997), suscita que numa nova perspectiva científica e metodológica é preciso ir da descrição para interpretação. Atentar conjuntamente a enfoques qualitativos e quantitativos para se obter a interpretação, tendo no corte etnográfico variáveis que auxiliam no entendimento da problemática ambiental. Assim sendo, estabelecer interpretações multicausais que aproxime da complexidade do objeto estudado.

Cabe ainda, considerar as interdependências enquanto conceito chave para a interpretação, pois não existe conflito isolado. O pesquisador deve relacionar com outros conflitos, encontrar os elementos de conexão, os fluxos e as formas que orientam o comportamento do sistema. Destarte, a relação de interdependência tem que considerar as relações Norte-Sul como chave para interpretação da problemática ambiental.

O papel do observador passa a ter uma maior importância, pois o mesmo é considerado parte da pesquisa, e por conta disso a subjetividade passa a fazer parte da construção do conhecimento e interpretação da realidade, da elaboração de hipóteses, no desenvolvimento da pesquisa e inclusive nos resultados. O sistema de acordo com Garcia (1994), se caracteriza pela sua vulnerabilidade ou resiliência. BIGGS et al. (2015, p. 2), descrevem a resiliência com base em sete princípios:
a) Princípio 1 - Manter a diversidade e redundância;
b) Princípio 2 - Gerenciamento da conectividade;
c) Princípio 3 - Gerenciamento de variáveis lentas e feebacks;
d) Princípio 4 - Adoção do pensamento sistemas adaptativos complexos;
e) Princípio 5 - Incentivar a aprendizagem;
f) Princípio 6 - Ampliar a participação;
g) Princípio 7 - Promover sistemas de governanças policêntricos.

Biggs et al. (2015), salientam a multiplicidade de fatores que fazem com que o sistema se torne resiliente. A ideia de ampliação dos sistemas de governança inserindo múltiplos atores e diversas formas organizacionais contém uma visão de que isso ajudaria a resguardar a utilização sustentável de um recurso. A diversidade ecológica entra como um valor importante, já que serve como meio de não haver maior dependência externa de recursos naturais. Explicitam que a conectividade, aspecto que aumenta o fluxo de informação pode tanto ter efeitos negativos quanto 
positivos, este último, em função da difusão de perturbações, bem como a capacidade de recuperação.

$\mathrm{O}$ incentivo à aprendizagem proposto vai além dos mecanismos criados dentro das universidades. Isso inclui a ampliação da participação, construindo relações de confiança, a fim de melhorar a legitimidade das propostas de intervenção. Para isso, é preciso criar um bom processo de participação com a escolha detalhada de seus participantes e ferramentas. A governança policêntrica auxilia no cumprimento de regras, na valorização do conhecimento tradicional e local e na partilha de conhecimento e aprendizagem.

García (1994), explica que a evolução dos sistemas possui mudanças escalares distintas entre subsistemas. Se há mudança escalar diferente dentro do sistema é preciso compreendê-las, dentro do que se configura a análise espacial. Estabelece uma crítica ao uso do termo totalidade em Marx, pois sua teoria não explicaria a relação entre a totalidade e as partes. O argumento: é preciso fazer um recorte da realidade mesmo em estudos sistêmicos. Surgem duas questões diante dessa constatação: ¿ es posible tomar en cuenta las interacciones que relacionan a dicho fragmento con la totalidad en la cual está inmerso sin que ello exija ampliar ad-infinitum los límites del estudio? (GARCÍA, 1994, p. 76). O autor responde fundamentando-se na "teoria geral dos sistemas" de Bartalanffy:

\footnotetext{
¿Pueden formularse bases conceptuales suficientes generales como para servir de marco a programas de investigación interdisciplinaria, es decir, que hagan posible un estudio que rebase los límites de disciplinas específicas, permitiendo un conocimiento integrado de problemas complejos de la realidad? (GARCÍA, 1994, p. 74).
}

Enquanto proposta metodológica recomenda-se realizar o diagnóstico, a fim de recuperar os principais processos que explicam o funcionamento do sistema. Assim adentra-se na fase de diferenciação e integração, a partir dos estudos da função e estrutura, com vista à compreensão do problema e a trazer prospecções para o planejamento. Nesse contexto, García (1994, p. 82), expõe a tese central de seu estudo los objetivos de uma investigación interdisciplinaria se logran a través del juego dialético em las fases de diferenciación e integración que tienen lugar en el proceso que condice a la definición y estúdio de um sistema complejo.

Como uma via para análise, Novo Villaverde (1997), propõe o modelo sistêmico, fundamentado na ideia de sistema de Bertlanlanffy (2010), no qual o meio ambiente pode ser estudado a partir de múltiplas interações, físico ou social. É preciso entender o sistema internamente, mas também o processo de organização que os mantém ligados ao ecossistema. $\mathrm{O}$ meio ambiente metodologicamente, enquanto instrumento de análises, segundo Novo Villaverde (1997), é visto como sistema de círculos concêntricos, em níveis de organização micro, meso e macro, que precisam ir à base de suas relações, na ideia de pensar globalmente e agir localmente.

O meio ambiente é visto como um sistema de valores, principalmente o modo de como a sociedade se relaciona com o ambiente depende dos valores éticos culturais de seu grupo. Novo Villaverde (1997), critica as visões antropocentristas e etnocentristas que tem regido o modo de atuação dos países desenvolvidos, pois não compreendem os limites da natureza e querem impor o modelo cultural ocidental ao resto do mundo. Situa que o modelo sistêmico é biocêntrico. Apresenta os grandes sistemas ambientais: biosfera; tecnosfera; sociosfera e noosfera e sugere que esses são sistemas complexos e dinâmicos, que atuam sobre equilíbrios dinâmicos ${ }^{6}$, operando sobre relações de ordem/mudanças e desordem/entropia.

Mesmo em contextos críticos, a partir de mecanismos de auto-organização, oriundos de estruturas dissipativas, os sistemas podem alcançar novo patamar de estabilidade. Portanto, o não equilíbrio é considerado como ordem, mas sim como um caminho para inovação, que converge

\footnotetext{
${ }^{6}$ A noção de equilíbrio dinâmico é uma das chaves para entender os problemas ambientais.
} 
para a ideia de unidade e diversidade, bem como de evolução indefinida "ningún sistema complejo es jamás estructuralmente estable" (NOVO VILLAVERDE, 1997, p. 39). Novo Villaverde (1997), propõe a metodologia interdisciplinar como sendo uma via para o conhecimento integrado - teoria e prática. Sugere trabalhar sobre relações, entre as partes e o todo com as partes. Relações estas que são intersistêmicas, com diferentes visões de mundo. Destarte, o enfoque sistêmico como modelo mental e a interdisciplinaridade como metodologia para lidar com a prática social e tecnológica.

$\mathrm{Na}$ prática, esses estudos devem auxiliar em propostas ambientalmente responsáveis e conduzir o sistema produtivo a um desenvolvimento sustentável, que inclui a melhoria da qualidade de vida das pessoas (GARCÍA, 1994). Numa perspectiva de proposição de alternativas regional esses estudos precisam ter caráter prospectivo, desde as condições iniciais até o desenvolvimento sustentável, prever os novos processos quando se introduz as mudanças, mas pensando sempre em um modelo melhor que o atual. Assumindo assim, um caráter de sistema reconstruído. A teoria sistêmica assinala e a investigação empírica confirma essas alterações nos sistemas. Destarte, a passagem de um estudo de diagnóstico para o estudo de políticas alternativas não é linear.

Como base da articulação interdisciplinar, Garcia (1994, p. 86), ou mesmo das revoluções científicas, coloca o marco epistêmico que seria al conjunto de preguntas, o cuestionamientos, no siempre formulados como preguntas precisas, que se plantea el investigador frente a un dominio de la realidad que se propone estudiar. Nele se apresenta a base de valores do pesquisador. Somente a partir desse "marco epistêmico" comum se pode pensar um estudo sistêmico que oriente a um diagnóstico integrado e a uma formulação compartilhada de políticas alternativas. O marco epistêmico seria então uma normatividad extradisciplinaría de contenido social (GARCIA, 1994, p. 87).

Enquanto metodologia, a interdisciplinaridade distingue-se da multidimensionalidade e da pluridimensionalidade pelo desenvolvimento da cooperação: "un proceso es interdisciplinar cuando en él se produce una cooperación articulada de diferentes perspectivas para la interpretación y/o resolución de cuestiones concretas, de orden intelectual o práctico" (NOVO VILLAVERDE, 1997, p. 42).

Novo Villaverde (1997), propõe o desenvolvimento de estratégias de isomorfismos conceituais e linguísticos ${ }^{7}$ a partir da cooperação e coesão de grupos humanos, em vista a configuração de uma metalinguagem, ou seja, do conhecimento integrado. A síntese interdisciplinar se daria então, através de um processo cíclico em que vários conceitos evoluem conjuntamente e conduzem a um novo padrão de inteligibilidade e de complexidade.

Si la interdisciplinariedad es un proceso de trabajo compartido en torno a información y lenguajes comunes, la transdisciplinaridad surge como producto cuando tal proceso tiene lugar en un marco de comprensión del mundo también compartido, de forma que se alcanza un metalenguaje y se integran teorías y prácticas bajo un mismo paradigma (NOVO VILLAVERDE, 1997, p. 46).

O autor indica que cabe a gestão ambiental assumir o papel de aplicar esses modelos e metodologias. $\mathrm{O}$ gestor tem que ser especialista e generalista, atuar a partir de uma cosmovisão, a fim de alcançar a síntese interdisciplinar. O papel da universidade seria o da internalização da educação superior, focos de compreensão não só interdisciplinar, mas também intercultural, de abertura a inovações externas. Um modo de cooperação entre universidades Norte/Sul, com base nas premissas da sustentabilidade, solidariedade e equidade. Com vistas a redimensionar, com base em um ponto de vista ambiental, os profissionais que a instituição forma.

\footnotetext{
${ }^{7}$ Para Novo Villaverde (1997) se apoiam na presença de principios gerais que se realizam e pode ser empregados em distintos sistemas (físicos, sociales, etc), a exemplo de totalidade e soma, feedback efeito umbral, entre otros.
} 
Destarte, García (1994), afirma que a concepção de investigação disciplinar é um importante instrumento de integração, seja na articulação dos estudos de grupos na prática concreta da investigação, seja pela interpretação da evolução de um sistema, como totalidade organizada na qual os diversos elementos estão em constante interação e onde se interconectam processos em distintas escalas espaciais e temporais. García (1994), apresenta algumas reflexões sobre a prática científica social, enfatizando a necessidade de mudança de enfoque no campo do meio ambiente, a exemplo dos estudos sobre a racionalidade ambiental, induzindo a melhoria do conhecimento sobre o processo de construção de valores, racionalidades e condutas, e o conflito de coexistência de diferentes racionalidades.

Garcia (1994), traça ainda algumas conclusões, a relação entre os problemas ambientais, a problemática geral, social e política. Alude sobre as mudanças nas formações sociais e naturais, que o investigador tenha uma tomada de consciência da dimensão social da ciência e da responsabilidade social do científico. Não transfere essa responsabilidade à ciência e nem a educação à distância. Destarte, reafirma as dificuldades epistemológicas e metodológicas dos estudos da problemática ambiental. Estas estão relacionadas com a fragmentação dos problemas, configurando-se como estudos setoriais, alicerçados por uma disciplina. Bem como, a escassez dos marcos conceituais que movem as disciplinas, necessitando reformular os enfoques tradicionais.

Emerge ainda a necessidade de se compreender os estudos complexos como uma totalidade, decorrente de domínios disciplinares diversos pela interação de elementos ou subsistemas. Por fim, confere aos programas de pós-graduação o lócus onde devem estar assentadas as bases concretas de investigações interdisciplinares. Esse é um desafio interdisciplinar que, segundo Novo Villaverde (1997), pode se ter o espelho da trajetória da educação ambiental na aplicação no ensino fundamental, pois encontra maior dificuldade no ambiente universitário. Propõe que este último esteja vinculado às necessidades reais de nosso tempo, a conjunção entre universidade e contexto.

Destarte, Novo Villaverde (1997), se coloca a favor do modelo de educação à distância, justificando que o docente passa a ser o facilitador de uma integração sincrônica entre o que se aprende e a realidade. Este vínculo entre pensamento e ação é atribuído à Educação Ambiental, no qual o autor o atribui a um conceito de tomada de decisão:

\footnotetext{
El proceso que consiste en acercar a las personas a un conocimiento complejo y global del medio ambiente (como un sistema de interacciones múltiples) para elucidar valores y desarrollar actitudes que les permitan adoptar una posición crítica y participativa sobre la conservación y correcta utilización de los recursos, la calidad de vida y el desarrollo sostenible (NOVO VILLAVERDE, 1997, p. 54).
}

Novo Villaverde (1997), explicita seu objeto de estudo, os modelos e metodologias de análises dos problemas ambientais. $\mathrm{O}$ autor apresenta de modo sistemático as inter-relações entre teoria e método, o pensamento sistêmico e a prática interdisciplinar, relacionando os principais pontos que abarcam a sua problemática de estudo. Didaticamente expõe o caráter sistêmico e interdisciplinar contido na Educação Ambiental, que ainda carece de ser explorado no ambiente acadêmico. Essa concepção não deve ser restringida à Educação Ambiental, mas as demais disciplinas e campos que não encontram na universidade o ambiente para o modelo de pensamento complexo possa ser exercitado.

Do mesmo modo que a educação à distância possa manter o vínculo com a realidade, o distanciamento provoca alterações nas interações e elucubrações mentais diferentes que o ambiente social da universidade oferece. Esse modelo de educação ainda é muito recente para falarmos em resultados orientados à interdisciplinaridade. O pensamento complexo apresenta elementos tantos modernos, quanto pós-modernos, pois insere o sujeito tanto como objeto, quanto 
observador, bem como, expõe as contradições entre "ordem/desordem, da parte/todo, do singular/geral" (BATISTA; SALVI, 2006, p. 152). Esse modo de ver as múltiplas relações intrínsecas aos objetos de estudos se aproxima da teoria do desenvolvimento histórico-geográfico desigual, que procura entender como o capital cria e se apropria da diversidade, a fim de atender seus interesses.

Enquanto justaposição metodológica procura ver as inter-relações entre as múltiplas escalas de atuação do capital e suas implicações geográficas. As convergências e divergências existentes entre os autores estão centradas nas noções de complexidade e de interdisciplinaridade. Enquanto Novo Villaverde (1997), compreende a complexidade sobre uma perspectiva biocêntrica, e aprofunda na teoria geral dos sistemas, Garcia (1994) irá aprofundar a análise epistemológica da teoria dos sistemas, mas centrado na sua dimensão espacial. Confere um papel importante à análise o processo, a função e a estrutura para se entender o sistema em sua totalidade.

Os processos envolvem escalas temporais que incidem de modo diverso nos subsistemas e produzem alterações nestes últimos. E a estrutura é o conjunto de relações que sedimenta a organização e que faz o sistema atuar em sua totalidade. A função é concebida como o aporte de cada elemento ou subsistema para o funcionamento do sistema. "Alude que a función y estructura (o entre proceso y estados) es la clave para comprensión de los fenómenos" (GARCIA, 1994, p. 133). Cabe destacar que Novo Villaverde (1997), também apresenta noções espaciais, quando institui o nível macro, meso e micro do sistema de círculos concêntricos.

De outro modo o aspecto da interdisciplinaridade apresenta elementos que coadunam. Garcia (1994) aborda a interdisciplinaridade enquanto metodologia, uma prática que precisa se aproximar da universidade, principalmente da pós-graduação, apresentando os caminhos para tal êxito, com base em marcos epistêmico, conceitual e metodológico compartilhados. Aspecto esse também corroborado por Novo Villaverde (1997), quando sugere a construção de uma metalinguagem.

Entretanto, quando Garcia (1994), alude mudanças nas formações sociais e naturais, recomenda que o investigador tenha uma tomada de consciência da dimensão social da ciência e da responsabilidade social do científico. Confere essa responsabilidade à ciência, mas não levanta a bandeira da educação à distância. Tanto o pensamento sistêmico, quando a metodologia interdisciplinar são o esteio para se pensar a questão do desenvolvimento geográfico desigual em sua relação com a configuração territorial em dada região. O pensamento sistêmico irá auxiliar no entendimento inter e intra sistemas escalares. A metodologia interdisciplinar nas formulações das hipóteses, dos marcos epistêmico, conceitual e propriamente metodológico de perquirição.

Destarte ambos os textos apresentam contribuições que se complementam, pois conferem uma ênfase no método explicativo, dirimindo os estudos estritamente descritivos ao abordar as questões que não só compete à problemática ambiental, mas que servem para o entendimento de sistemas sociais, como é o caso dos sistemas produtivos regionalizados e sistemas de inovação nacional/regional.

\section{OS SISTEMAS DE INOVAÇÃO REGIONAL}

Conceitos como distritos industriais (BECATTINI, 1990; BRUSCO, 1990), clusters (PORTER, 1990), meios inovadores (CAMAGNI, 1991), sistemas regionais de inovação (COOKE, 2001) e regiões de aprendizagem (ASHEIM, 1996), sublinharam a importância das regiões como motores essenciais da inovação (ASHEIM; BOSCHMA; COOKE, 2011). O estudo dos processos de inovação a partir da configuração territorial conforma duas abordagens principais que dialogam, são elas: os sistemas nacionais de inovação e os sistemas regionais de inovação. Seu entendimento requer uma análise multiescalar da interação dos diversos atores. O que alude ao seu caráter complexo. 
Cooke et al. (1997), estabelecem uma relação entre os sistemas nacionais de inovação (SNI) com os sistemas regionais de inovação (SRI). Estes argumentam que o aspecto escalar subnacional, delimitado em âmbito regional, torna mais factível a pesquisa e agrega conteúdo relevante para entender o liame entre região, sistema e inovação. Justificam que o estudo nacional dos processos de inovação torna-se complexo. Ressaltam os aspectos atrelados à capacidade institucional nos SRI: o financeiro, a aprendizagem e as culturas produtivas. Expressam ainda que existem diferentes modos de inovação regional, descritos em sua maioria pelos fluxos de conhecimento e informação e pelo modo que interagem seus atores. Criticam o curto prazo da expectativa em relação à política de inovação.

Explicam que a política de inovação envolve ora regionalismos, ora regionalização. A regionalização é capaz de dotar a região de uma política de inovação autônoma e orientar recursos financeiros em prol do sistema regional. O regionalismo em função de aspectos culturais da capacidade produtiva oportuniza uma possível atuação sistêmica. O modelo de inovação sistêmica proposto por Cooke et al. (1997), fundamenta-se na vantagem competitiva, na qual empresas estabelecem cooperação institucionalizada. Assim constituem-se as regiões de aprendizagem e clusters regionais inovadores, com vantagem competitiva assentada em inovação. Uma aprendizagem bidirecional proveniente da interação com outros lugares, no qual inovação e aprendizagem estão relacionadas.

Lundvall et al. (2002), reiteram a importância dos SNI, e sua aplicação deve ser adaptada nos países subdesenvolvidos, por isso têm por objetivo adaptar a aplicação de sistemas nacionais de inovação, um conceito largamente utilizado nos países ao Norte. Explicam que na OCDE as altas taxas de crescimento estão relacionadas à formação de diferentes sistemas de investigação nos países. Deste modo, a escala nacional continua a ser importante para processos de inovação. Os casos analisados colocam o modelo Dinamarquês do Aalborg como versão referência para os estudos de sistemas nacionais de inovação, haja vista que este combina crescimento econômico, igualdade, indústria primária, baixa tecnologia e flexibilidade com segurança laboral.

Diferente do modelo dos EUA, vinculado à alta tecnologia, que atrela crescimento com aumento da desigualdade e pode ser justificado por seus sistemas de produção especializados na produção de máquinas-ferramentas, o que permite uma flexibilização da produção, traduzido também como flexibilização laboral. O modelo Aalborg é uma versão que se centra na visão de longo prazo; no estabelecimento da confiança, elemento central para o desenvolvimento das capacidades interativas de aprendizagem e inovação; e uma racionalidade comunicativa, permitindo a coordenação e cooperação para inovação do produto. Tal conceito é inspirado em Schumpeter, na conformação de uma nova teoria do crescimento; e da economia de aprendizagem, que inclui indivíduos, organizações e regiões. Assim, os processos de inovação dependem da interação e criatividade humana, das atividades de produção e do cenário institucional, e devendo ser observado além do âmbito nacional, na perspectiva local/regional.

Sua aplicação no Sul requer a superação das desigualdades, o desenvolvimento de ações políticas em busca da construção do sistema. Um modelo visto como capaz de promover o crescimento econômico sustentável e do bem-estar. No entanto, deve-se atentar para a relação de poder das redes globais de conhecimento intensivo e sua capacidade de incluir e excluir no processo de desenvolvimento do Estado-nação. Freeman (2002) vai ampliar a abordagem trazendo a escala continental para análise, para além das já mencionadas nacional e subnacional. Questiona o papel dos sistemas de inovação na determinação das variações de taxas de crescimento entre regiões.

Perpassa sua análise para a Grã-Bretanha no século XVIII, os Estados Unidos a partir da metade do século XIX, e dos países desenvolvidos no século XX. Evidencia a problemática em inovação a complementariedade entre ciência, tecnologia, economia, política e cultura e de SNI e SRI. No caso do sistema britânico há um papel central da ciência e da indústria, e de uma interação 
entre ciência, tecnologia, cultura e empreendedorismo. Denota a forte atuação do SNI diante do SRI com forte complementariedade entre eles. Destaca a relevância dos retornos crescente de escala e das economias de aglomeração do modelo britânico. Parafraseando Schumpeter ressalta que a inovação em sua gênese é desigual em relação ao aspecto temporal e espacial.

Expõe que o SNI dos Estados Unidos inicialmente se assemelhava do modelo britânico. Avançou pelas condições ambientais, agregada ao desenvolvimento tecnológico e investimento científico, com apoio à inovação técnica e a ênfase na ciência e na tecnologia financiada pelo Estado, gerando novas indústrias. Cabe destacar as economias de escala e os efeitos para frente e para trás das indústrias e o alto custo de investimento em inovação, que para os países subdesenvolvidos a imitação é a alternativa. Crítica a teoria da convergência, da visão de vantagem locacionais junto a países líderes, a exemplo do Japão no século XX e sua influência no desenvolvimento econômico dos países asiáticos. Destaca ainda os investimentos em inovação e processos de aprendizagem na Coreia do Sul e em Taiwan, e o aumento no número de patentes.

Sintetiza que o crescimento subnacional se configura de modo desigual e que os sistemas nacionais de inovação, de mudanças tecnológicas e institucionais servem também para explicar as lacunas existentes entre países desenvolvidos e subdesenvolvidos no seu crescimento econômico. Freeman (1995), já destacava a importância dos SNI e SRI para análise econômica e afirma que possuem perspectiva histórica, e é sustentada pela Nova Teoria do Crescimento. Destaca o papel do sistema nacional de educação, das relações industriais, principalmente a crescente conexão internacional e das instituições técnicas, científicas, e das políticas governamentais e tradições culturais.

Relaciona no caso da Alemanha o êxito do sistema nacional de inovação a investimento em ciência, educação e tecnologia, este último vinculado à máquina-ferramenta. Explica que a pesquisa e desenvolvimento, originalmente denominado Research \& Development (R\&D), afeta significativamente os SNI, e as empresas transnacionais têm um papel central nesse processo. O avanço institucional se deu na Alemanha. Nas décadas de 1970 e 1980 ficou evidente o papel das instituições nacionais para manutenção dos SNI, que inclui países como os Estados Unidos, Japão e da Europa. Destarte a inovação técnica específica dependia de alterações no sistema de produção.

A abordagem sistêmica se consolida com o advento das novas tecnologias (tecnologia da informação, biotecnologia e tecnologia de novos materiais). Freeman (1995), destaca que a diferença do sucesso japonês para o caso Soviético é que o primeiro aplicava em R\&D para a economia civil e não para a guerra como no segundo caso. Além disso, o SNI na União Soviética não era integrado. Relaciona o êxito do crescimento econômico de ambos na década de 1950 e 1960 ao investimento em educação e a ênfase na ciência e na tecnologia.

Freeman (1995), salienta ainda que os casos analisados apontam para o desenvolvimento desigual da economia mundial e de diferentes taxas de crescimento e profundas diferenças entre os SNI das nações em função de mudanças institucionais e técnicas, gerando características qualitativas de análise e que o uso dos métodos quantitativos da teoria neoclássica não dá conta dessa realidade. Expõe que há um discurso que coloca a ideia de que as transnacionais via globalização estão mudando a economia mundial e quebrando as barreiras espaciais do EstadoNação. Daí o surgimento de abordagens supranacionais ou subnacionais, sustentado em Porter (1990). O autor explica a vantagem competitiva criada de modo localizado, no país de origem das empresas.

Destarte, é preciso cautela, pois essas empresas amoldam-se às características locais com o uso racional da diversidade, mas mantém o controle e os investimentos em P\&D no âmbito doméstico, por isso a necessidade de controle dessa inter-relação nos sistemas nacionais. Além disso, ocorre acumulação de capital proveniente do comércio internacional que é reinvestido no país, explicitado pelo aumento do fosso entre os países industrializados e subdesenvolvidos. É aquilo que Harvey (2013), vai chamar de um novo imperialismo. Destaca o papel dos blocos 
econômicos e as entidades subnacionais como um conjunto hierarquicamente organizado em que confluem processos de acumulação de capital.

\section{CONSIDERAÇÕES FINAIS}

A contribuição da teoria dos sistemas, do pensamento complexo e da abordagem interdisciplinar no que tange aos sistemas de inovação, revela-se de suma importância. A teoria dos sistemas auxilia no entendimento das unidades e as alterações escalares (micro, meso e macro) entre subsistemas que compõe os sistemas de inovação, suas relações entre si, o que caracteriza sua vulnerabilidade ou resiliência, o modo como se organiza e sua tendência para a integração de seus elementos concretos e abstratos, tendo assim uma função dentro do sistema, seja ele regional ou nacional.

No entanto, o recorte disciplinar oferecido na análise das unidades não é suficiente, requer um aprofundamento das relações entre si, no tocante ao contexto que gere uma abordagem interdisciplinar, que privilegie o jogo dialético das fases de diferenciação e integração do sistema em seus aspectos físicos ou sociais, tal qual prevalecem os estudos na área de desenvolvimento regional que propiciam o conhecimento integrado. Assim sendo, a abordagem interdisciplinar propicia ir além de estudos descritivos, já que ocorre uma necessidade de construção de conhecimento e interpretação da realidade que de todo modo se apresenta complexa, com múltiplos atores, não cabendo, portanto, uma resposta no campo disciplinar, já que este enfoque é limitante para lidar com problemas locais circunscritos que são concretos, territorializados e de caráter multicausal.

Destarte, o pensamento complexo compreende as heterogeneidades presentes nos sistemas de inovação e sua relação de interdependência, o que impede uma análise setorial e localista dos sistemas de inovação, cabendo, portanto, compreender os sistemas de inovação como um objeto de estudo a ser perquirido entre disciplinas, pois os sistemas agem de modo integrado, em múltiplas escalas e não são estruturalmente estáveis. Deste modo, a região emerge como uma dimensão escalar importante para compreender os processos de sistemas de inovação. Isso dá-se em função de correlações entre aspectos territoriais de mudanças institucionais e técnicas, e as imbricações entre região, sistema e inovação.

A região torna-se mais favorável à pesquisa e agrega outros elementos. Um deles é a aprendizagem bidirecional a partir da interação com outros lugares. No entanto, as experiências apresentadas sobre sistemas de inovação refletem a realidade dos países ao Norte em melhor contexto de desenvolvimento que os países ao Sul. Por isso, devem ser analisadas como referenciais epistemológicos apenas para suscitar questionamentos e possibilidades diante do contexto dos países ao Sul. Pois, há indicações que para atingir tais resultados é preciso superar as desigualdades, exigindo políticas orientadas para a constituição do sistema.

\section{REFERÊNCIAS}

ASHEIM, B. T.; BOSCHMA, R.; COOKE, P. Constructing regional advantage: Platform policies based on related variety and differentiated knowledge bases. Regional Studies, v. 45, n. 7, p. 893-904, 2011. Disponível em: https://www.tandfonline.com/doi/full/10.1080/00343404.2010.543126?casa_token=fpyPuC4HCVEAAA AA\%3AzP4qyn1Nxbo3bFFBC8XpOsjNLxH7RVqL2iJoDP8RoXHC3ZGkW3Q81nJStQTfdd8116XqG91 YeXKxu_s. Acesso em: 06 ago. 2020.

ASHEIM, B. T.; GERTLER, M. S. The geography of innovation: regional innovation systems. In: The Oxford handbook of innovation, 2009. 
ASHEIM, B. Industrial districts as learning regions: a condition for prosperity. European Planning Studies, 4(4): 379-400. 1996. Disponível em: https://www.tandfonline.com/doi/abs/10.1080/09654319608720354. Acesso em: 06 ago. 2020.

BATISTA, I. L.; SALVI, R. F. Perspectiva pós-moderna e interdisciplinaridade Educativa: pensamento complexo e reconciliação integrativa. Ensaio, vol. 8, n. 2, p.147-159, 2006. Disponível em: https://www.scielo.br/scielo.php?pid=S1983-

21172006000200171\&script=sci_arttext\&tlng=pt>. Acesso em: 06 ago. 2020.

BECATTINI, G. The Marshallian industrial district as a socio-economic notion. In Industrial Districts \& Inter-firm Co-operation in Italy, Edited

by: Pyke, P., Becattini, G. and Sengenberger, W. 37-51. Geneva: International Institute for Labour Studies (ILO). 1990.

BERTALANFFY, L. V. Teoria geral dos sistemas: fundamentos, desenvolvimento e aplicações. Petrópolis: Vozes, $5^{\mathrm{a}}$ ed, p. 311, 2010.

BIGGS, R.; SCHÜTER, M.; SCHOON, M. L. Principles for building resilience: sustaining ecosystem services in social-ecological Systems. Cambridge: Cambrigdge University Press, 2015.

BRUSCO, S. The idea of the industrial district: its genesis. In Industrial Districts \& Inter-firm Co-operation in Italy, Edited by: Pyke, F., Becattini, G. and Sengenberger, W. 10-

19. Geneva: International Institute for Labour Studies (ILO). 1990.

CAMAGNI, R. Innovation Networks: Spatial Perspectives. Edited by: Camagni, R. London: Belhaven. 1991.

CAPRA, F.; LUISI, P. L. A visão sistêmica da vida: uma concepção unificada e suas implicações filosóficas, políticas, sociais e econômicas. São Paulo: Cultrix, 2014.

CHRISTOFOLETTI, A. Análise de sistemas em geografia. São Paulo: Hucitec-Edusp, p.106, 1979.

COOKE, P. et al. (Ed.). Handbook of regional innovation and growth. Edward ElgarPublishing, 2011.

COOKE, P.; URANGA, M. G.; ETXEBARRIA, G. Regional innovation systems: Institutional and organizational dimensions. Research Policy, vol. 26, p. 475-491, 1997. Disponível em: https://www.sciencedirect.com/science/article/abs/pii/S0048733397000255. Acesso em: 06 ago. 2020.

COOKE, P. Regional innovation systems, clusters, and the knowledge economy. Industrial and Corporate Change, 10(4): 945-974. 2001. Disponível em: https://academic.oup.com/icc/article-abstract/10/4/945/706922. Acesso em: 06 ago. 2020.

DAVIDSON, M. Uncommon sense. The life and thought of Ludwig von Bertalanffy, father of General Systems Theory. Boston: J.P. Tarcher, INC, p.238, 1983. 
DIAS, M. F. P.; PEDROZO, E. A.; DA SILVA, T. N. The innovation process as a complex structure with multilevel rules. Journal of Evolutionary Economics, v. 24, n. 5, p. 1067-1084, 2014. Disponível em <https://link.springer.com/article/10.1007/s00191-014-0384-2>. Acesso em: 06 ago. 2020.

FREEMAN, C. Continental, national and sub-national innovation systems - complementarity and economic growth. Research Policy, vol.31, p.191-211, 2002. Disponível em: https://www.sciencedirect.com/science/article/pii/S0048733301001366?casa_token=GSzv13Sdo BYAAAAA:kimikh_JUM8gLgYDVfqjmtljU1EJv0yQSwq45MImasuHW2OGgoQAGbctH1edn2HH0OFx7pZxDtl. Acesso em: 06 ago. 2020.

FREEMAN, C. The national system of innovation in historical perspective. Cambridge Journal of Economics, vol.19, p.5-24, 1995. Disponível em: https://academic.oup.com/cje/articleabstract/19/1/5/1708372. Acesso em: 06 ago. 2020.

GARCÍA, R. Interdisciplinaridad y sistemas complejos. Revista Latinoamericana de Metodología de las Ciencias Sociales, v. 1, n. ,. p. 66- 101, 2011.

GARUD, R.; GEHMAN, J.; KUMARASWAMY, A. Complexity arrangements for sustained innovation: Lessons from 3M Corporation. Organization Studies, v. 32, n. 6, p. 737-767, 2011. Disponível em: https://journals.sagepub.com/doi/abs/10.1177/0170840611410810. Acesso em: 06 ago. 2020.

GIL, A. C. Como elaborar projetos de pesquisa. (6. ed.) São Paulo: Atlas. 2017.

HARVEY, D. O novo imperialismo. $7^{\mathrm{a}}$ ed. São Paulo: Edições Loyola, 2013.

LUNDVALL, B. Å.; JOHNSON, B.; ANDERSEN, E. S. A.; DALUM, B. National systems of production, innovation and competence building. Research Policy, vol. 31, p. 213-231, 2002. Disponível em:

https://www.sciencedirect.com/science/article/pii/S0048733301001378?casa_token=0My2W3Sc Ej0AAAAA:cAxwjAuF8xKSdgINqPOvNxvikF60EctMEFiWEO5_pIdjV974lhmRByKqBb3vm Diykh-133_NGBYK. Acesso em: 06 ago. 2020.

NOVO VILLAVERDE, M. El análisis de los problemas ambientales: modelos y metodología, In: NOVO, M.; LARA, R. (Orgs). El análisis interdisciplinar de la problemática ambiental. Madrid: UNESCO, p.21-59, 1997.

PORTER, M. The Competitive Advantage of Nations. London: Macmillan. 1990.

SCHOT, J.; STEINMUELLER, W. E. Three frames for innovation policy: R\&D, systems of innovation and transformative change. Research Policy, v. 47, n. 9, p. 1554-1567, 2018. Disponível em: https://www.sciencedirect.com/science/article/pii/S0048733318301987. Acesso em: 06 ago. 2020.

SCHUMPETER, J. A. The Theory of economic development. Harvard Univ. Press, Cambridge, 2004. 
URIONA-MALDONADO, M.; DOS SANTOS, R. N. M; VARVAKIS, G. State of the art on the Systems of Innovation research: a bibliometrics study up to 2009. Scientometrics, v. 91, n. 3, p. 977-996, 2012. Disponível em: https://akjournals.com/view/journals/11192/91/3/articlep977.xml. Acesso em: 06 ago. 2020.

VALE, C. C. Teoria geral do sistema: histórico e correlações com a geografia e com o estudo da paisagem. Entre-Lugar, v. 3, n. 6, p. 85-108, 2012. Disponível em:

http://ojs.ufgd.edu.br/index.php/entre-lugar/article/view/2448. Acesso em: 06 ago. 2020.

VAN DE VEM, A. H.; POLLEY, D. E.; GARUD, R. V. S. The innovation journey. Oxford University Press, New York, 2007.

Recebido: $22 / 02 / 2021$

Aceito: $20 / 10 / 2021$

Publicado: Dezembro de 2021 